\title{
Society of Neurolnterventional Surgery Standards of Practice: general considerations
}

\author{
P M Meyers, ${ }^{1}$ K A Blackham, ${ }^{2}$ T A Abruzzo, ${ }^{3}$ C D Gandhi, ${ }^{4}$ R T Higashida, ${ }^{5}$ \\ J A Hirsch, ${ }^{6} \mathrm{C}$ J Moran, ${ }^{7} \mathrm{~S}$ Narayanan, ${ }^{8} \mathrm{C}$ J Prestigiacomo, ${ }^{9} \mathrm{R}$ Tarr, ${ }^{3}$ On behalf of \\ the Society for Neurolnterventional Surgery
}

\begin{abstract}
${ }^{1}$ Department of Radiology and Neurological Surgery, Columbia University, College of Physicians and Surgeons, and

Neuroendovascular Service, New York

Presbyterian-Columbia,

Neurological Institute of New

York, New York, USA

${ }^{2}$ University Hospitals Case

Medical Center, Cleveland, Ohio,

USA

${ }^{3}$ University of Cincinnati Medical Center, Cincinnati, Ohio, USA

${ }^{4}$ UMDNJ-New Jersey Medical

School, Newark, New Jersey,

USA

${ }^{5}$ University of California San

Francisco Medical Center, San Francisco, California, USA

${ }^{6}$ Massachusetts General Hospital, Boston,

Massachusetts, USA

${ }^{7}$ Mallinkrodt Institute of

Radiology, St Louis, Missouri,

USA

${ }^{8}$ Department of Neurosurgery and Neurology, Wayne State University/Detroit Medical

Center, Detroit, Michigan, USA

${ }^{9}$ University of Medicine and

Dentistry of New Jersey,

Newark, New Jersey, USA
\end{abstract}

\section{Correspondence to}

Dr P M Meyers, Department of

Radiology and Neurological

Surgery, Columbia University,

College of Physicians and

Surgeons, and

Neuroendovascular Service

New York

Presbyterian-Columbia,

Neurological Institute of New York, 710 West 168th Street, Rm 428, New York, New York 10032, USA;

pmm2002@columbia.edu

Accepted 1 November 2011

\section{ABSTRACT}

This is the first in a set of documents intended to standardize techniques, procedures, and practices in the field of endovascular surgical neuroradiology. Standards are meant to define core practices for peer review, comparison, and improvement. Standards and guidelines also form the basic dialogue, reporting, and recommendations for ongoing practices and future development.

\section{INTRODUCTION}

\section{Purpose, rationale and importance}

This document is intended to standardize techniques, procedures and practices in the field of endovascular surgical neuroradiology. This is important, not only to improve healthcare outcomes but also to define the core practice on which this specialty can build and grow. Innovation must continue; however, our core practice should be clarified and codified to create a stable source for peer review, comparison and improvement. Standards and guidelines are also important to form the basic language for dialog and reporting and to establish the reference from which recommendations for future investigation can be made.

\section{Intended audience and targeted health problem}

Without these fundamentals and definitions, evidence based reporting is obscured by idiosyncratic details and incomparable statistics. The practice of endovascular surgical neuroradiology has changed dramatically and rapidly in the past 15 years and therefore a systematic review, analysis and translation of the abundance of clinical and basic science research into meaningful clinical practice parameters is critical. The Society of NeuroInterventional Surgery (SNIS) is in a key position to lead such an endeavor, as a national society representing members from a variety of training backgrounds which include interventional neuroradiologists, endovascular neurosurgeons and interventional neurologists, who are now devoted to the subspecialty practice of neurointerventional surgery. Nevertheless, all endovascular subspecialty practitioners and minimally invasive spine practitioners will benefit from this wide ranging project of integration of current best evidence and clinical expertise such as can be provided by SNIS. The patient population served by endovascular surgical neuroradiology includes those affected by intracranial, extracranial, spinal, head and neck vascular malformations, tumors and atherosclerotic disease, as well as acute ischemic stroke. ${ }^{1}$

\section{Definitions and methods of practice parameters}

The purview of each forthcoming standard of practice will discuss, at a minimum: (1) the clinical criteria for diagnosis and treatment of a particular disease entity and the expected health outcomes, (2) a rigorous review of the literature for peer reviewed evidence, (3) a discussion of the diagnostic and therapeutic options, including data for efficacy and safety and (4) recommendations for their use utilizing the evidence as classified by the quality of study design. The evidence will be classified by American College of Cardiology/American Heart Association Clinical Practice Guidelines, a commonly used and widely accepted system. ${ }^{2} 3$ Recommendations will be defined. The method of literature review will be stated in each standard document. Ideally, the standards will be reviewed and renewed every 5 years.

\section{Writing group composition}

The practice parameters are produced by the Standards of Practice Committee of the SNIS, which includes a broad range of research, clinical expertise and experience in neurointerventional surgery. The standards will be reviewed and approved by the executive board of the SNIS, or its designated committees.

\section{PHYSICIAN QUALIFICATIONS}

From its inception, qualifications to perform endovascular neurological interventions have been a somewhat controversial issue. This may be due to the comparative novelty of neurointervention which came into existence only relatively recently in the past 20 years, the paucity of evidence based data early on compared with standard neurosurgical outcomes and practices, and the lack of well trained practitioners until training standards were recently approved in 2001. ${ }^{5}$ Physicians with neuroscience training are not the only medical specialists drawn to this field. Physician groups with little or no formal training in neurological disease diagnosis or management have expressed the desire to perform neurointerventional treatment procedures, such as interventional cardiologists performing carotid and intracranial stenting, and acute ischemic stroke therapy. This trend goes against the specialization of medical disciplines that occurred over the past half century. While certain specialties have explicitly described the 
importance of cognitive and procedural training, many of these groups do not share the same concerns about neurological diseases and organ specific medical training. In certain cases, physicians who argue for the importance of specialization in their own disciplines, simultaneously argue against specialization in the neurosciences, thus generating inconsistencies that are difficult to reconcile.

Within the neurosciences, there is a basic set of training standards for neurointervention that is recognized by the Accreditation Council for Graduate Medical Education. ${ }^{5}$ There is tremendous variability between neurointerventional training programs reflecting different practice patterns and other factors. The SNIS is committed to maintain and advance these criteria as standard bearers for the discipline. Standards of practice are an important step towards achievement of the goal-generalized acceptance and recognition of neurointerventional techniques in routine medical practice with patient safety and procedural efficacy to improve patient outcomes and overall healthcare. ${ }^{4-9}$

\section{GENERAL PRINCIPLES}

This document provides general principles of performance, qualifications, preparation, intraprocedure and postprocedure care, as applied to procedures performed by the neurointerventional surgical physician. These considerations should be applicable to the individual disease processes and procedures discussed in each of the following sections which are divided by specific disease processes (box 1). General considerations are discussed separately to diminish redundancy that might be created by discussing them in the context of each chapter. However, more detailed consideration will be included in each individual chapter, where applicable.

\section{QUALIFICATIONS}

The operating neurointerventionist should have appropriate training and experience, and meet the qualifications for performance of neuroangiography and neurovascular intervention. The neurointerventionalist should adhere and satisfy the SNIS guidelines for performance of neuroangiography which have been published previously. ${ }^{10}$ The neurointerventionalist should have full and complete knowledge about the indications, risks, expectations, benefits, alternatives, advantages, disadvantages and technical details of each procedure. The neurointerventionalist must be familiar with and capable of performing rescue interventions for known or anticipatable potential complications. The neurointerventionalist must have

\section{Box 1 Guidelines and standards section documents}

- Acute ischemic stroke and treatment

- Arteriovenous malformation and treatment

- Balloon test occlusion

- Carotid stenosis and treatment

- Cerebral artery aneurysm and treatment

- External carotid artery embolization

- Head and neck chemotherapy

- Head and neck tumor embolization

- Intracranial atherosclerotic disease and treatment

- Spinal artery embolization

- Vasospasm and treatment

- Vertebral augmentation a thorough understanding of extracranial and intracranial vascular anatomy (including congenital and developmental variants and common collateral pathways), angiographic equipment, radiation safety and physiological monitoring equipment. The neurointerventionalist must have available all necessary supplies, devices, x-ray monitoring equipment and personnel (nurses, technologists, anesthesiologists) to safely perform these procedures. Finally, the neurointerventionalist must understand the principles of complication avoidance (including prevention of thromboembolic phenomena), perioperative hydration, antithrombotic and anticoagulant therapy, puncture site hemostasis and management of neuroangiographic complications.

The neurointerventionalist must be able to understand the clinical significance of angiographic abnormalities and unsuspected findings. The ability to accurately interpret angiographic images obtained during the procedure, as well as an understanding of non-invasive imaging (MRI, MR angiography, CT and CT angiography), prior to the procedure is necessary. Image interpretation is not a casual byproduct of the procedure but an intrinsic requirement that directs the 'real time' conduct of any procedure. The neurointerventionalist should have a full understanding of the disease process and alternative methods of treatment. The basic and advanced techniques used in neurointerventional procedures should reflect the principles espoused in the training guidelines of the Accreditation Council on Graduate Medical Education program in Endovascular Surgical Neuroradiology. ${ }^{4}$

\section{RADIOGRAPHIC IMAGING EQUIPMENT AND STAFF}

Biplane digital subtraction cerebral angiography, flat panel or high resolution image intensifier and imaging chain (preferably 1024 X 1024 matrix or greater) is highly recommended. Road map fluoroscopy is mandatory for many procedures, preferably with simultaneous real time unsubtracted fluoroscopic imaging. The ability to document fluoroscopy time and estimated dosimetry is important and now mandatory. Staff requirements include certified radiologic technologists who are trained to operate angiographic equipment as well as medical physicists who can evaluate the equipment performance initially and annually, and provide expert advice on image quality issues and radiation safety.

\section{MATERIALS}

It is often not possible to predict which embolic materials, catheters or guidewires will be required to treat a specific disease process. Therefore, multiple sizes and types of guiding catheters, guidewires, balloon catheters, microcatheters and microguidewires, and a full complement of embolic materials, must be maintained in the inventory or available on short notice. Most products are now available on consignment to hospitals from the manufacturers.

\section{PATIENT SELECTION AND PREPARATION}

The indications for treatment, rationale, informed consent and alternatives to therapy should be documented in the patient's medical record. This should include all relevant clinical (as documented in the history and physical examination) and radiological criteria (as documented in the pretreatment cross sectional imaging and diagnostic angiography). The potential need for specialized neuroscience critical care, neuroanesthesia and neurosurgical consultation should be taken into consideration in preoperative planning. The neurointerventional 
procedure represents only one part of the treatment paradigm for many patients with neurovascular disease. To ensure the best possible neurological outcomes, all necessary equipment and expertise should be available at the treatment center.

A well documented medical history which includes a complete medical history of the presenting symptoms, indications for the procedure, pertinent medical and surgical histories, list of current medications, history of allergies (especially allergies to nickel and iodinated contrast), and vascular and neurologic risk factors needs to be documented.

A physical examination should be performed before and after treatment, including a detailed neurologic examination. The preoperative neurological examination is necessary to establish the proper treatment indications, plan the treatment procedure, monitor the response to treatment (and anesthetic agents) and differentiate pre-existing from procedure related deficits. The examination should be comprehensive with special detail to the involved vascular territory at risk, and to assist in planning the angiographic approach. In patients with lesions involving the visual pathways, baseline perimetry testing of the visual fields should be considered. In patients with lesions of the hippocampus or other limbic structures, preoperative neuropsychological testing should be useful.

Appropriate and complete diagnostic neuroangiography should precede any form of intervention. The results of this study should be permanently recorded and should be of sufficient detail to characterize the extent of vascular disease that might contribute to the patient's clinical syndrome, treatment effects and post-treatment clinical course. This frequently requires angiographic study of adjacent and remote vessels that contribute to the regional collateral circulation. Diagnostic angiography may be performed at the same time as the intervention, and represents an intention to treat paradigm, if appropriate patient counseling has already been performed, and if consent for treatment has been obtained. In all cases, postprocedural 'control' arteriography should also be performed to permanently record and to document the anatomic result(s) and to identify potential complications. Images obtained should be available to consulting physicians for review using a picture archiving and communication system.

Informed patient consent should be obtained in all cases, unless imminently life threatening circumstances require immediate emergent treatment. Informed consent should include the indications for treatment, technical nature of the procedure, discussion of the potential alternative treatments (including no interventional treatment, medical management, surgical resection or occlusion and radiotherapy), discomforts, benefits and risks of the procedure. Radiation risks vary among neurointerventional procedures, and these should be discussed with the patient according to local, state and National Council for Radiation Protection (NCRP) guidelines. The consent process and discussion should be charted in the medical record. Any off-label application of drugs or devices should be described therein.

Laboratory evaluation is warranted prior to cerebrovascular neurointervention. When anesthesia or nurse sedation services will be used, institutional standards for preoperative evaluation will guide this particular practice. At a minimum, basic laboratory testing prior to catheter angiography typically includes testing hemoglobin, hematocrit, platelet count, creatinine, electrolyte levels, coagulation parameters and electrocardiography. In women of childbearing age, a current pregnancy test should be documented. Patients taking anticoagulant or antithrombotic medications may require preoperative analysis of blood type (eg, type and screen or type and cross match) for rapid transfusion in the event of serious bleeding complications.

\section{PROCEDURAL CARE}

Generally, diagnostic catheter angiography is performed with some form of analgesia and sedation according to institutional standards for moderate sedation, and interventional treatment procedures are frequently performed with either monitored or general anesthesia according to local institutional standards. All patients should undergo continuous cardiac and intermittent blood pressure monitoring during the procedure. Invasive blood pressure monitoring may be appropriate or necessary in certain situations where intraprocedure and postprocedure measurements of blood pressure are critical. If the patient is to be sedated in any form, pulse oximetry should also be monitored. A record of vital signs should be maintained. All patients should have appropriate intravenous access for the administration of fluids and medications as needed. Where heparin is to be administered, activated clotting time measurements should be available. While not a standard of care, patients taking antithrombotic agents, including aspirin or clopidogrel or other antiplatelet agent, may be evaluated using aggregometry (eg, VerifyNow; Accumetrics, San Diego, California, USA).

Equipment and medications for emergent resuscitation should be immediately available, and all personnel present should be trained in their use according to American Heart Association guidelines for basic life support and advanced cardiac life support. Equipment, pharmaceuticals and physician expertise to manage angiographic and interventional complications, including cerebral thromboembolic complications, should be available. Where the risk of a procedural intracranial hemorrhage exists, a ventriculostomy kit should be readily available.

A record of materials used, and all device implants, should be documented in the procedure note. Fluoroscopy times and estimated patient exposure should be recorded in the medical record. Any significant exposure should prompt review and patient counseling. ${ }^{11}$ All potentially exposed hospital personnel should be monitored for radiation exposure on a monthly, quarterly and annual basis.

Techniques used for pediatric patients may require attention to certain procedural details that are different from those commonly encountered during treatment of adults. Modification of adult standards for neurointerventional procedures, if necessary, will depend on the age of the patient and the clinical condition for which treatment is performed. Specific considerations include, but are not limited to, indications, informed consent from parents or legal guardians, anesthesia, periprocedural monitoring, and contrast and radiation dose.

Specific positioning of anesthesia machines and monitors within the neurointerventional suite must permit unrestricted access to the patient throughout the procedure. The use of general anesthesia may preclude clinical and neurologic examination of the patient but the advantages usually outweigh such concerns, especially during intracranial or spinal vascular interventions. When patients are under general anesthesia, the use of electrophysiological monitoring may achieve an increased level of safety. When feasible, sedation, rather than general anesthesia, may be advantageous to permit neurologic examination throughout the procedure. For procedures such as carotid stent revascularization or provocative anesthetic testing prior to embolization or arteriovenous malformations, sedation can be useful.

Because nearly all pediatric interventional procedures require general anesthesia, close coordination with the pediatric 
anesthesiology service is necessary for safe performance. The development of hypothermia, particularly in infants, should be anticipated and guarded against by the use of temperature monitors, control of ambient room temperature, warming blankets, drapes and head coverings. Fluid balance must be monitored to prevent overhydration as a result of flush solutions or osmotic dehydration from iodinated contrast injection. Additional hemodynamic monitoring may be appropriate, particularly in the setting of cardiac failure or when blood pressure manipulation is necessary during the procedure. Contrast dose must be kept to the minimum amount that is consistent with effective performance of the procedure and recorded. Minimal diagnostic injections and dilution of contrast medium for digital angiography should be used. If clinically appropriate, staging of extensive procedures may be considered to mitigate the potential adverse effects of the iodinated contrast load and radiation exposure.

\section{POSTPROCEDURAL CARE}

All patients should be observed and bed rest continued for $6-24 \mathrm{~h}$ during the initial postprocedural period for a duration dependent on the size of the arteriotomy and the use of arteriotomy closure devices. Extended periods of bed rest may be necessary in the presence of indwelling devices. Neurological intensive care is now a specific critical care discipline. Many comprehensive stroke centers are developing competence in this discipline and may represent the optimal level of clinical care for neurointerventional patients. During the initial postprocedural period, skilled nurses or other trained professionals should periodically monitor the puncture site for hematoma development and distal peripheral pulses. Monitoring of indwelling arterial sheaths to assure continuous flushing should be performed. Given end organ vulnerability, neurointerventional procedures may require ongoing anticoagulant or antithrombotic medications. Neurological monitoring is important to detect delayed neurological deterioration.

Postprocedure monitoring should be conducted in a manner appropriate to the disease process and anatomic lesion treated. The patient should be monitored for urinary output, renal function, cardiac symptoms, pain and other indicators of systemic complications. Further laboratory evaluation of hematologic, coagulation or organ function may be indicated. Appropriate follow-up imaging (skull x-rays, CT, MR, angiography, Doppler blood flow) should be obtained when indicated.

The initial ambulation of the patient must be supervised. The operating physician or designee should evaluate the patient's condition after the procedure. Patient evaluations should be documented in the medical record. The operating physician or designee must remain available for continuing care during hospitalization and following discharge.

Thresholds for appropriateness, successes and neurologic complications are individually discussed in each chapter by disease process. Thresholds for puncture site complications, preliminary planning neuroangiography complications and contrast induced nephropathy may be greater than those suggested for diagnostic neuroangiography. ${ }^{10}$

\section{RADIATION PROTECTION}

Fluoroscopic imaging continues to be the mainstay of endovascular surgical neuroradiology. It has long been assumed that the radiation exposure necessary to complete a procedure is justified by the disease state for which the patient is undergoing treatment. This concept is captured in the ALARA (as low as reasonably achievable) principle, which states that exposure to ionizing radiation must be kept as low as possible considering relevant societal and economic factors. ${ }^{12}$ Societal and economic factors shape the ALARA principle when use of alternative procedures is not indicated by virtue of the reduction in radiation exposure that would be achieved. However, the cost, complexity and duration of neurointerventional procedures could reach a point that alternative therapeutic methods would be favored. It is the responsibility of each neurointerventionalist to consider these issues and for the neurointerventional community to actively participate in the assessment and resolution of these issues.

Neurointerventional procedures commonly fall into the category of high exposure fluoroscopic procedures. When more than 30 min of fluoroscopy are necessary to complete a procedure, the kerma area product, $\mathrm{P}_{\mathrm{KA}}$, of at least $300 \mathrm{~Gy} \mathrm{~cm}^{2}$ has likely been reached. Such procedures warrant concern: each procedure should be justified according to the medical goal accomplished, and patient follow-up to evaluate for radiation injury is necessary. These procedures must be performed on appropriately configured and calibrated equipment, and the equipment used must have dose monitoring capability that is recorded in a rigorous fashion.

According to NCRP guidelines, radiation exposure of patients is specifically unlimited but must be justified, while occupation exposure to healthcare personnel is limited. Both must be actively monitored and recorded. Most fluoroscopy units have a timer mechanism that records total fluoroscopy time. Fluoroscopy time is not a measurement of patient exposure. Measurements of kerma area product $\left(\mathrm{P}_{\mathrm{KA}}\right)$ and total air kerma $\left(\mathrm{K}_{\mathrm{a}, \mathrm{r}}\right)$ are used to compare fluoroscopically guided procedures. Effective dose (E) is a surrogate marker for stochastic radiation risk, for comparison of fluoroscopically guided procedures, and for institutional review board analysis of possible stochastic radiation risk to patients and populations. Peak dose $\left(\mathrm{D}_{\text {tissue, max }}\right)$ is used to describe deterministic radiation effects to tissue, such as skin or ocular lens. Moreover, procedural risks of radiation exposure must be included and specifically reviewed in any procedural consent.

The neurointerventionalist should receive formal training in radiation physics, biology and safety. The interventionalist must be knowledgeable about radiation physics and dose management to receive fluoroscopy privileges and to appropriately control patient exposure during procedures. In all cases, the neurointerventionalist is responsible for the radiation exposure generated and for the exposure to the patient and the medical staff. Patient dose should be recorded in the medical record after each exposure in the event that any single exposure or cumulative exposure becomes a significant dose, requiring counseling, according to NCRP guidelines. Personnel dosimetry must be used for staff that is present during fluoroscopy. The practitioner must be aware of and compliant with, state regulations regarding use of the equipment and licensing of users. In some locations, local (municipal and/or county) requirements apply in addition to state rules. Practitioners should participate in radiation control regulatory matters in consultation with his/her qualified medical physicist and the facility's radiation safety officer.

\section{CONCLUSION}

Building on these general principles, the topical documents that follow contain a review of the best level of evidence at the time of writing and a compendium of recommended practice standards. With improvements in technology applicable to treatment of patients with neurological disease, these guidelines may 
change as the challenges we faced yesterday evolve into those we face tomorrow.

Acknowledgments The authors would like to thank and acknowledge all members of the SNIS Executive Committee for their review and endorsement of this guidelines document.

\section{Competing interests None.}

Provenance and peer review Commissioned; not externally peer reviewed.

\section{REFERENCES}

1. Hirsch JA, Meyers PM, Barr J, et al. Technical standards and practice guidelines: should we? Why now? Why SNIS? J Neurointerv Surg 2009:1:5-7.

2. Gibbons RJ, Smith S, Antman E; American College of Cardiology; American Heart Association. American College of Cardiology/American Heart Association clinical practice guidelines: Part I: where do they come from? Circulation 2003;107:2979-86.

3. Gibbons RJ, Smith SC Jr, Antman E; American College of Cardiology; American Heart Association. American College of Cardiology/American Heart Association clinical practice guidelines: Part II: evolutionary changes in a continuous quality improvement project. Circulation 2003:107:3101-7.

4. Higashida RT, Hopkins LN, Berenstein A, et al. Program requirements for residency/ fellowship education in neuroendovascular surgery/interventional neuroradiology: a special report on graduate medical education. AJNR Am J Neuroradiol 2000;21:1153-9

5. ACGME Program Requirements for Graduate Medical Education in Endovacular Surgical Neuroradiology. 2008. http://www.acgme.org/acWebsite/downloads/ RRC_progReq/182_endovascular_neuroradiology_01012008_u06102008.pdf (accessed 29 0ct 2011).

6. Higashida RT, Furlan AJ, Roberts $\mathrm{H}$, et al. Trial design and reporting standards for intra-arterial cerebral thrombolysis for acute ischemic stroke. Stroke 2003;34:e109-37.

7. Higashida RT, Meyers PM, Phatouros CC, et al. Reporting standards for carotid artery angioplasty and stent placement. Stroke 2004;35:e112-34.

8. Meyers PM, Schumacher HC, Higashida RT, et al. Reporting standards for endovascular repair of saccular intracranial cerebral aneurysms. J Neurointerv Surg 2010;2:312-23.

9. Schumacher HC, Meyers PM, Higashida RT, et al. Reporting standards for angioplasty and stent-assisted angioplasty for intracranial atherosclerosis. Stroke 2009; 40:e348-65.

10. Quality improvement guidelines for adult diagnostic neuroangiography. Cooperative study between the ASNR, ASITN, and the SCVIR. American Society of Neuroradiology. American Society of Interventional and Therapeutic Neuroradiology. Society of Cardiovascular and Interventional Radiology. AJNR Am J Neuroradiol 2000:21:146-50.

11. Klein LW, Miller DL, Balter S, et al. Occupational health hazards in the interventional laboratory: time for a safer environment. Radiology 2009:250:538-44.

12. Hendee WR, Edwards FM. ALARA and an integrated approach to radiation protection. Semin Nucl Med 1986:16:142-50.

Advancing Postgraduates. Enhancing Healthcare.

The Postgraduate Medical Journal is dedicated to advancing the understanding of postgraduate medical education and training.

- Acquire the necessary skills to deliver the highest possible standards of patient care

- Develop suitable training programmes for your trainees

- Maintain high standards after training ends

Published on behalf of the fellowship for Postgraduate Medicine

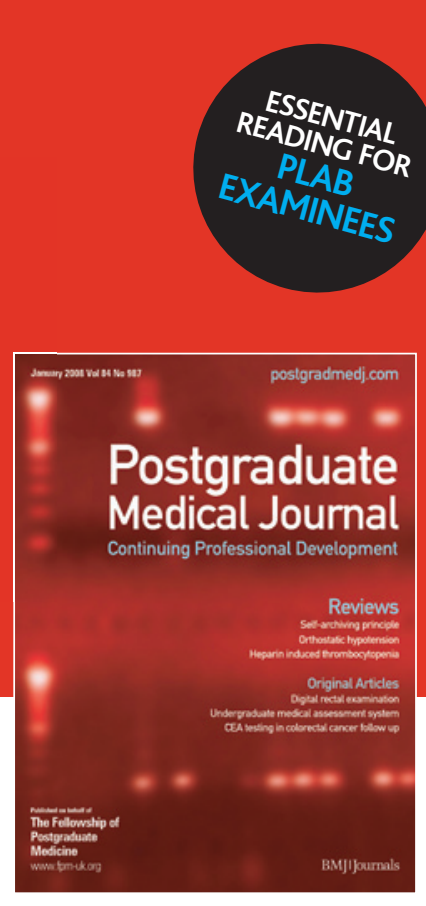

FOR MORE DETAILS OR TO SUBSCRIBE, VISIT THE WEBSITE TODAY 


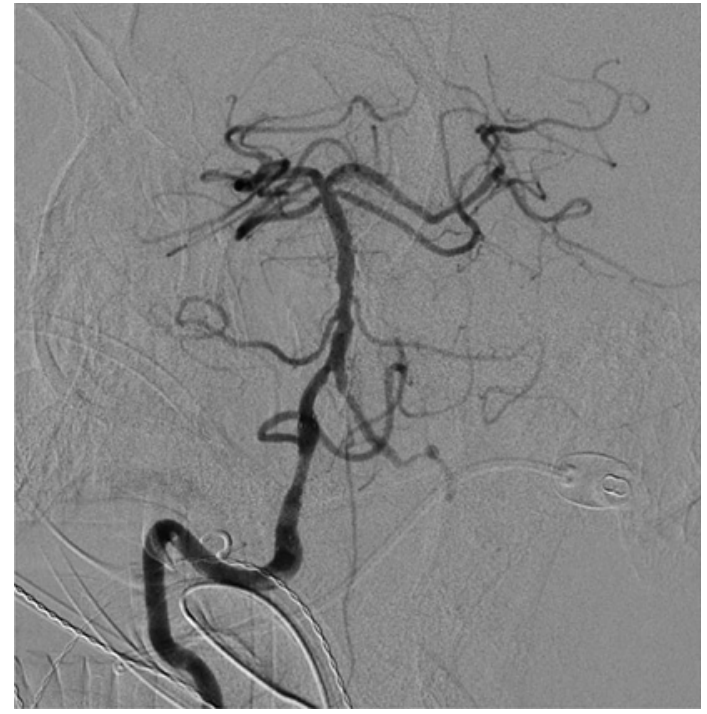

Figure 2 Post-angioplasty and stenting angiogram shows recanalization of the right vertebral artery with significantly improved flow through the posterior circulation with normalization of flow through the anterior spinal artery.
On catheter angiography, the intracranial vertebral artery segments and the proximal basilar artery were only supplied by retrograde flow through the anterior spinal artery (figure 1B). Right and left carotid angiograms did not show posterior communicating arteries. However, there was slow, delayed, retrograde flow into the left posterior cerebral artery and the top of the basilar artery via pial collaterals from the middle cerebral artery to the posterior cerebral artery (figure 1C). The patient underwent successful right vertebral artery stenting resulting in markedly improved flow through the posterior circulation (figure 2). Following the procedure the patient's neurological exam significantly improved. Upon discharge to a rehabilitation facility, the patient was awake, alert and following all commands. The patient was able to move all limbs against gravity with some resistance.

Competing interests None.

Provenance and peer review Not commissioned; externally peer reviewed.

\section{Corrections}

Meyers PM, Blackham KA, Abruzzo TA, Gandhi CD, Higashida RT, Hirsch JA, Moran CJ, Narayanan S, Prestigiacomo CJ, Tarr R, on behalf of the Society for NeuroInterventional Surgery. Society of NeuroInterventional Surgery Standards of Practice: general considerations J NeuroIntervent Surg 2012;4:11-15. doi:10.1136/neurintsurg-2011-010180

The author list has been updated to read:

Meyers PM, Blackham KA, Abruzzo TA, Gandhi CD, Higashida RT, Hirsch JA, Hsu D, Moran CJ, Narayanan S, Prestigiacomo CJ, Tarr R, on behalf of the Society for NeuroInterventional Surgery.

J Neurointervent Surg 2012;4:115. doi:10.1136/neurintsurg-2011-010180corr1

Eicker S, Etminan N, Turowski B, et al. Intracranial carotid artery stent placement causes delayed severe intracranial hemorrhage in a patient with moyamoya disease $J$ NeuroIntervent Surg 2011;3:160-2. doi:10.1136/jnis.2010.003004

This article was published with the incorrect doi number. The correct doi number is: doi:10.1136/jnis.2010.3004

J Neurointervent Surg 2012;4:115. doi:10.1136/jnis.2010.3004corr1 
7. Alaraj A, Wallace A, Mander N, et al. Outcome following symptomatic cerebral vasospasm on presentation in aneurysmal subarachnoid hemorrhage: coiling vs clipping. World Neurosurg 2010;74:138-42.

8. Wikholom G, Lindgren $\mathrm{H}$, Rodriguez $\mathrm{H}$, et al. Embolization with Guglielmi detachable coils during the period of increased risk for cerebral vasospasm: early outcome. Neuroradiology 2000:42:833-7.

9. Murayama Y, Malisch T, Guglielmi G, et al. Incidence of cerebral vasospasm after endovascular treatment of acutely ruptured aneurysms: report on 69 cases. $J$ Neurosurg 1997:87:830-5.

10. Morizane A, Nakahara I, Sakai N, et al. Endovascular surgery for treated aneurysm with symptomatic vasospasm. No Shinkei Geka 1999;27:941-6.

11. Murayama Y, Song JK, Uda K, et al. Combined endovascular treatment for both intracranial aneurysm and symptomatic vasospasm. AJNR Am J Neuroradiol 2003;24:133-9

12. Sugiu K, Katsumata A, Ono $\mathrm{Y}$, et al. Angioplasty and coiling of ruptured aneurysm with symptomatic vasospasm: technical case report. Surg Neurol 2003;59:413-17.

13. Tosaka M, Okajima F, Hashiba Y, et al. Sphingosine 1-phosphate contracts canine basilar arteries in vitro and vivo. Possible role in pathogenesis of cerebral vasospasms. Stroke 2001;32:2913-19.

14. Shibuya M, Suzuki Y, Sugita K, et al. Effect of AT877 on cerebral vasospasm after aneurismal hemorrhage. J Neurosurg 1992;76:572-7.

15. Kassell NF, Helm G, Simmons N, et al. Treatment of cerebral vasospasm with intraarterial papaverine. J Neurosurg 1992;77:848-52.

16. Wanke I, Dorfler A, Dietrich U, et al. Combined endovascular therapy of ruptured aneurysms and cerebral vasospasm. Neuroradiology 2000;42:926-9.

17. Hope JKA, Byrne JV, Molyneux AJ, et al. Factors influencing successful angiographic occlusion of aneurysms treated by coil embolization. AJNR Am J Neuroradiol 1999;20:391-9.

18. Byrne JV. Acute endovascular treatment by coil embolization of ruptured intracranial aneurysms. Ann R Coll Surg Engl 2001;83:253-7.

19. Kaku Y, Yonekawa Y, Tsukahara T, et al. Superselective intra-arterial infusion of papaverine for the treatment of cerebral vasospasm after subarachnoid hemorrhage. J Neurosurg 1992;77:842-7.
20. Terada T, Kinoshita $\mathrm{Y}$, Yokote $\mathrm{H}$, et al. The effect of endovascular therapy for cerebral arterial spasm, its limitations and pitfalls. Acta Neurochir (Wien) 1997; 139:227-34.

21. Clouston JE, Numaguchi $Y$, Zoarski GH, et al. Intraarterial papaverine infusion for cerebral vasospasm after subarachnoid hemorrhage. AJNR Am J Neuroradiol 1995:16:27-38.

22. Jin $\mathbf{Y}$, Sagher 0 , Thai $\mathrm{QA}$, et al. The effects of papaverine on phorbol dibutylate-induced vasoconstriction in brain slice microvessels. J Neurosurg 1994;81:574-8.

23. Clyde BL, Firlik AD, Kaufmann AM, et al. Paradoxical aggravation of vasospasm with papaverine infusion following aneurysmal subarachnoid hemorrhage. J Neurosurg 1996:84:690-5.

24. Biondi A, Ricciardi GK, Puybasset L, et al. Intra-arterial nimodipine for the treatment of symptomatic cerebral vasospasm after aneurysmal subarachnoid hemorrhage: preliminary results. AJNR Am J Neuroradiol 2004;25:1067-76.

25. Mayer TE, Dichagans M, Straube A, et al. Continuous intra-arterial nimodipine for the treatment of cerebral vasospasm. Cardiovasc Intervent Radiol 2008;31:1200-4.

26. Keuskamp J, Murali R, Chao KH, et al. High-dose intraarterial verapamil in the treatment of cerebral vasospasm after aneurysmal subarachnoid hemorrhage. $J$ Neurosurg 2008;108:458-63.

27. Albanese $\mathbf{E}$, Russo $A$, Quiroga $M$, et al. Ultrahigh-dose intraarterial verapamil in the treatment of cerebral vasospasm after aneurysmal subarachnoid hemorrhage. $J$ Neurosurg 2010:113:913-22.

28. Tachibana E, Harada T, Shibuya M, et al. Intra-arterial infusion of fasudil hydrochroride for treating vasospasm following subarachnoid haemorrhage. Acta Neurochirurgica (Wien) 1999;141:13-19.

29. Hanggi D, Beseoglu K, Turowski B, et al. Feasibility and safety of intrathecal nimodipine on posthaemorrhagic cerebral vasospasm refractory to medical therapy and endovascular therapy. Clin Neurol Neurosurg 2008;110:784-90.

30. Eskridge JM, McAuliffe W, Song JK, et al. Balloon angioplasty for the treatment of vasospasm: results of first 50 cases. Neurosurgery 1998;42:510-17.

31. Eskridge JM, Song JK, Elliott JP, et al. Balloon angioplasty of the A1 segment of the anterior cerebral artery narrowed by vasospasm. J Neurosurg 1999:91:153-6.

\section{Corrections}

Blackham KA, Meyers PM, Abruzzo TA, et al. Endovascular therapy of acute ischemic stroke: report of the Standards of Practice Committee of the Society of NeuroInterventional Surgery. J NeuroIntervent Surg 2012;4:87-93.

The following author's details should read: Albuquerque FC, Barrow Neurosurgical Associates LTD, Phoenix, AZ, USA.

J Neurolntervent Surg 2012:4:195. doi:10.1136/neurintsurg-2011-010243corr1

Meyers PM, Blackham KA, Abruzzo TA, Gandhi CD, Higashida RT, Hirsch JA, Hsu D, Moran CJ, Narayanan S, Prestigiacomo CJ, Tarr R. on behalf of the Society for NeuroInterventional Surgery. Society of NeuroInterventional Surgery Standards of Practice: general considerations. J NeuroIntervent Surg 2012;4:11-15. doi:10.1136/neurintsurg-2011010180 .

The following author has been added to the author list: Dr. Muhammad Shazam Hussein

J Neurolntervent Surg 2012:4:195. doi:10.1136/neurintsurg-2011-010180corr2

Eicker S, Etminan N, Turowski B, et al. Intracranial carotid artery stent placement causes delayed severe intracranial hemorrhage in a patient with moyamoya disease. J NeuroIntervent Surg 2011;3:160-162. doi:10.1136/jnis.2010.003004.

This article was published with the incorrect doi number. The correct doi number is: doi:10.1136/jnis.2010.3004.

J Neurolntervent Surg 2012:4:195. doi:10.1136/jnis.2010.3004 